Canadian

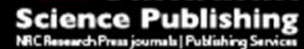

Canadian Journal of Chemistry Revue canadienne de chimie

\title{
Topological indices of rhombus type silicate and oxide networks
}

\begin{tabular}{|c|c|}
\hline Journal: & Canadian Journal of Chemistry \\
\hline Manuscript ID & cjc-2016-0486.R1 \\
\hline Manuscript Type: & Article \\
\hline Date Submitted by the Author: & 27-Oct-2016 \\
\hline Complete List of Authors: & $\begin{array}{l}\text { Javaid, Muhammad; University of Science and Technology of China (USTC), } \\
\text { Hefei, Anhui 230026, P.R.China.,, School of Mathematical Sciences } \\
\text { Rahman, Masood; University of Science and Technology of China (USTC), } \\
\text { Hefei, Anhui 230026, P.R.China., School of Mathematical Sciences, } \\
\text { Cao, Jinde; Southeast University, Nanjing, Jiangsu 210096, P.R. China., } \\
\text { Department of Mathematics }\end{array}$ \\
\hline Keyword: & Topological indices, rhombus silicate network and rhombus oxide network. \\
\hline
\end{tabular}

SCHOLARONE ${ }^{m}$

Manuscripts 


\title{
Topological indices of rhombus type silicate and oxide networks
}

\author{
M. Javaid ${ }^{a *}$ Masood Ur Rehman ${ }^{a}$, Jinde $\mathrm{Cao}^{b}$ \\ ${ }^{a}$ School of Mathematical Sciences, University of \\ Science and Technology of China (USTC), \\ Hefei, Anhui, P.R.China 230026. \\ ${ }^{b}$ Department of Mathematics, Southeast University, \\ Nanjing, Jiangsu, P.R. China 210096. \\ javaidmath@gmail.com \\ masood@mail.ustc.edu.cn \\ jdcao@seu.edu.cn
}

${ }^{*}$ Corresponding Author: M. Javaid, Email; javaidmath@gmail.com, School of Mathematical Sciences, University of Science and Technology of China (USTC) Hefei, Anhui, P.R. China. 


\begin{abstract}
For a molecular graph, a numeric quantity which characterizes the whole structure of a graph is called a topological index. In the studies of quantitative structure-activity relationship (QSAR) and quantitative structure-property relationship (QSPR), topological indices are utilized to guess the bioactivity of chemical compounds. In this paper, we compute general Randić, first general Zagreb, generalized Zagreb, multiplicative Zagreb, atom-bond connectivity (ABC) and geometric-arithmetic (GA) indices for the rhombus silicate and rhombus oxide networks. In addition, we also compute the latest developed topological indices such as the fourth version of $\mathrm{ABC}$ $\left(A B C_{4}\right)$, the fifth version of GA $\left(G A_{5}\right)$, augmented Zagreb and Sanskruti indices for the foresaid networks. At the end, a comparison between all the indices is included and the result is shown with the help of a cartesian coordinate system.
\end{abstract}

Keywords: Topological indices, rhombus silicate network and rhombus oxide network. 


\section{Introduction}

The combination of chemistry, mathematics and information science leads to a new subject called by cheminformatics. It studies the quantitative structure-activity relationship (QSAR) and the quantitative structure- property relationship (QSPR) that are used to predict the biological activities and properties of chemical compounds. The physico-chemical properties and the topological indices play an important role in the studies of QSAR and QSPR.

Graph theory has found a considerable use in a branch of mathematical chemistry which is known as chemical graph theory. It applies graph theory to the mathematical model of chemical phenomenon. Computing of the topological indices is a subtopic of chemical graph theory, which correlates certain physico-chemical properties of the underlying chemical compound. Simply, we can say that the topological indices are designed by transforming a molecular graph into a number and these are invariant under graph isomorphisms. There are some main classes of topological indices such as distance based topological indices, degree based topological indices and polynomials related indices of graphs. Among these classes degree based topological indices are of great importance and play a vital role in chemical graph theory and particularly in chemistry.

In 1947, Wiener first time made the use of a topological index in chemistry working on boiling point of paraffin [13]. He introduced the notion of path 
number of a graph as the sum of distances between any two carbon atoms in the molecules, in terms of carbon-carbon bonds which later called by the name of Wiener index. In graph theoretical language, the Wiener index is equal to the count of all shortest distances in a graph. After the Wiener index the theory of topological indices is started. In the progressive studies of indices, a large number of topological indices has been introduced by different chemist, and many researchers have proved different computing and theocratical results related to the certain topological indices for different families of molecular graphs.

In particular, Rajan et al. [7] computed the Randić, Zagreb and atombond connectivity indices of silicate $(S L(n))$, honeycomb $(H C(n))$ and hexagonal $(H X(n))$ networks. Recently, Baig et al. [2] calculated Randić, atombond connectivity, geometric-arithmetic, general zagreb, forth atombond connectivity and fifth geometric-arithmetic indices for dominating oxide $(D O X(n))$, dominating silicate $(D S L(n))$, and regular triangulene oxide $(\operatorname{RTOX}(n))$ networks. In this paper, we compute general Randić, first general Zagreb, generalized Zagreb, multiplicative Zagreb, atom-bond connectivity $(\mathrm{ABC})$ and geometric-arithmetic (GA), the fourth version of $\mathrm{ABC}\left(A B C_{4}\right)$, the fifth version of GA $\left(G A_{5}\right)$, augmented Zagreb and Sanskruti indices for the rhombus silicate $(R H S L(n))$ and rhombus oxide $(\operatorname{RHOX}(n))$ networks. For further studies of the topological indices, we refer, $[1,3,4,6,19,20,25]$.

The rest of the paper is organised as, Section 2 includes the definitions 
and formulas which are frequently used in the main results. In Section 3, we compute the main results related to the topological indices of rhombus silicate networks. Section 4 includes the main results on topological indices for the rhombus oxide networks, and in Section 5, we compare the results of all the indices and draw the conclusion.

\section{Preliminaries}

A molecular graph $\Gamma=(V(\Gamma), E(\Gamma))$ with the vertex set $V(\Gamma)=\left\{v_{1}, v_{2}, \ldots\right.$, $\left.v_{n}\right\}$ and the edge set $E(\Gamma)$ is a graph whose vertices denote atoms and edges denote bonds between that atoms of any underlying chemical structure. The order and size of a graph is $|V(\Gamma)|=v$ and $|E(\Gamma)|=e$ respectively. A graph is connected if there exists a connection between any pair of vertices. A loop is an edge that connects a vertex to itself and two or more than two edges with same end points are multiple edges. A network is simply a finite connected graph having no multiple edges and loops. The distance between two vertices $u$ and $v$ denoted by $d(u, v)$ is the length of the shortest path between $u$ and $v$ in graph $\Gamma$. The length of the shortest path between $u$ and $v$ is also called a $u-v$ geodesic. The longest path between any two vertices is called a $u-v$ detour. The degree of a vertex $v$ denoted by $d(v)$ is the number of vertices that are connected to $v$ by the edges. Moreover, we define $S_{u}=\sum_{v \in N_{\Gamma}(u)}(d(v))$ and $S_{v}=\sum_{u \in N_{\Gamma}(v)}(d(u))$, where $N_{\Gamma}(u)=\{v \in V(\Gamma) \mid u v \in E(\Gamma)\}$ and $N_{\Gamma}(v)=\{u \in V(\Gamma) \mid u v \in E(\Gamma)\}$.

Now, we define certain topological indices which will be used frequently in 
the main results. We begin from Wiener index which is defined in 1947.

Definition 2.1. [13] Let $\Gamma$ be a graph and $(u, v)$ be any ordered pair of its vertices with $d(u, v)$ as a $u-v$ geodesic. Then, the Wiener index of $\Gamma$ is defined as

$$
W(\Gamma)=\frac{1}{2} \sum_{(u, v)} d(u, v)
$$

In 1972, Gutman and Trinajsti [14] derived a pair of molecular descriptors known as the first Zagreb index and the second Zagreb index for the total $\pi$-energy of conjugated molecules. Soon after these indices have been used as branching indices [16, 24]. Later the Zagreb indices found applications in QSPR and QSAR studies [15, 17].

Definition 2.2. Let $\Gamma$ be a molecular graph. Then, the first Zagreb and the second Zagreb indices denoted by $M_{1}(\Gamma)$ and $M_{2}(\Gamma)$ respectively are defined as

$$
\begin{aligned}
& M_{1}(\Gamma)=\sum_{u v \in E(\Gamma)}[d(u)+d(v)], \\
& M_{2}(\Gamma)=\sum_{u v \in E(\Gamma)}[d(u) \times d(v)] .
\end{aligned}
$$

In 1975, Milan Randić [23] defined the following degree based topological index which is called Randić index.

Definition 2.3. Let $\Gamma$ be a molecular graph. Then, the Randić index 
denoted by $R_{-\frac{1}{2}}(\Gamma)$ is defined as

$$
R_{-\frac{1}{2}}(\Gamma)=\sum_{u v \in E(\Gamma)} \frac{1}{\sqrt{d(u) \times d(v)}}
$$

In 1998, Bollobás and Erdös [5], and Amic et al. [8] independently generalized the concept of Randić index and established the generalized Randić index. It has been extensively studied by both theoretical chemists and mathematicians. By the way, many important properties and results are established. To know in details, we refer [12, 27, 28].

Definition 2.4. Let $\Gamma$ be a molecular graph. Then, for a real number $\alpha$, the general Randić index denoted by $R_{\alpha}(\Gamma)$ is defined as

$$
R_{\alpha}(\Gamma)=\sum_{u v \in E(\Gamma)}[d(u) \times d(v)]^{\alpha} .
$$

Thus, for $\alpha=-\frac{1}{2}, \alpha=\frac{1}{2}$ and $\alpha=1$, then the general Randic index is a Randić index, reciprocal Randić index and the second Zagreb index respectively. For further study of the general Randić index, we refer [12, 28, 27].

Li and Zheng (2005)[26] defined the first general Zagrab index as follows.

Definition 2.5. Let $\Gamma$ be a molecular graph. Then, for a real number $\alpha$ the first general Zagrab index denoted by $M_{1}^{\alpha}$ is defined as

$$
M_{1}^{\alpha}=\sum_{u \in V(\Gamma)}[d(u)]^{\alpha}
$$


In 2011, Iranmanesh and Azari [18] introduced the generalized Zagreb index based on degree of vertices of a connected graph $\Gamma$.

Definition 2.6. Let $\Gamma$ be a molecular graph. Then, for $r, s \in Z^{+}$, the generalized Zagreb index is denoted by $M_{r, s}(\Gamma)$ and defined as

$$
M_{r, s}(\Gamma)=\sum_{u v \in E(\Gamma)}\left[d(u)^{r} d(v)^{s}+d(v)^{r} d(u)^{s}\right]
$$

In 2012, Ghorbani and Azimi [21] defined the new multiplicative versions of $M_{1}(\Gamma)$ and $M_{2}(\Gamma)$ as follows.

Definition 2.7. Let $\Gamma$ be a molecular graph. Then, the first multiplicative Zagreb index and the second multiplicative Zagreb index denoted by $P M_{1}(\Gamma)$ and $P M_{2}(\Gamma)$ are defined as

$$
\begin{aligned}
& P M_{1}(\Gamma)=\prod_{u v \in E(\Gamma)}(d(u)+d(v)), \\
& P M_{2}(\Gamma)=\prod_{u v \in E(\Gamma)}(d(u) \times d(v)) .
\end{aligned}
$$

In 1998, Estrada et al.[10] introduced the atom bond connectivity index which has been applied to study the stability of alkanes and the strain energy of cycloalkanes. Later on, Ghorbani and Hosseinzadeh (2010) [22] defined the forth version of atom bond connectivity index.

Definition 2.8. Let $\Gamma$ be a molecular graph. Then, the atom bond connectivity index and the forth version of atom bond connectivity index denoted 
by $A B C(\Gamma)$ and $A B C_{4}(\Gamma)$ respectively are defined as

$$
\begin{gathered}
A B C(\Gamma)=\sum_{u v \in E(\Gamma)} \sqrt{\frac{d(u)+d(v)-2}{d(u) \times d(v)}}, \\
A B C_{4}(\Gamma)=\sum_{u v \in E(\Gamma)} \sqrt{\frac{S(u)+S(v)-2}{S(u) \times S(v)}} .
\end{gathered}
$$

In 2009, Vukičević and Furtula [9] defined the geometric-arithmetic $(G A)$ index. Later on the fifth version of the geometric-arithmetic $\left(G A_{5}\right)$ index is defined by Graovac et al. (2011) [1].

Definition 2.9. Let $\Gamma$ be a molecular graph. Then, the geometricarithmetic index and the fifth version of the geometric-arithmetic index denoted by $G A(\Gamma)$ and $G A_{5}(\Gamma)$ respectively are defined as

$$
\begin{gathered}
G A(\Gamma)=\sum_{u v \in E(\Gamma)} \frac{2 \sqrt{d(u) \times d(v)}}{d(u)+d(v)}, \\
G A_{5}(\Gamma)=\sum_{u v \in E(\Gamma)} \frac{2 \sqrt{S(u) \times S(v)}}{S(u)+S(v)} .
\end{gathered}
$$

In 2010, Furtula et al. [6] proposed the modified version of the ABC index called by augmented Zagreb index and recently Sunilkumar M. Hosamani (2016) defined the Sanskruti index motivated by the augmented Zagreb index.

Definition 2.10. Let $\Gamma$ be a molecular graph. Then, the augmented Zagreb index and the Sanskruti index denoted by $A Z I(\Gamma)$ and $S(\Gamma)$ re- 
spectively are defined as

$$
\begin{gathered}
A Z I(\Gamma)=\sum_{u v \in E(\Gamma)}\left[\frac{d(u) \times d(v)}{d(u)+d(v)-2}\right]^{3}, \\
S(\Gamma)=\sum_{u v \in E(\Gamma)}\left[\frac{S(u) \times S(v)}{S(u)+S(v)-2}\right]^{3} .
\end{gathered}
$$

Now, we discuss the construction of rhombus silicate and rhombus oxide networks. The most interesting class of minerals by far is silicate. These are obtained by fusing metal oxides or metal carbonates with sand. Essentially all the silicates contain $\mathrm{SiO}_{4}$ tetrahedra as it is a basic unit. In chemistry, the corner vertices of $\mathrm{SiO}_{4}$ tetrahedron represent oxygen ions and the center vertex represents the silicon ion. In graph theory, we call the corner vertices as oxygen nodes and the center vertex as silicon node. By the different arrangements of the tetrahedron silicate, we can obtain different silicate structures. Similarly, different silicate networks are constructed by different silicate structures. In Figure 1, the rhombus silicate network of dimension 3 is presented. In general, the vertices and the edges in a rhombus silicate of dimension $n$ are $5 n^{2}+2 n$ and $12 n^{2}$, respectively. If we delete all the silicon ions from the rhombus silicate network, then we obtain rhombus oxide network as shown in Figure 2 of dimension 3. For dimension $n$, the vertices and the edges of a rhombus oxide network are $3 n^{2}+2 n$ and $6 n^{2}$ respectively, where dimension means the number of vertices existing in any side of the rhombus silicate or oxide network. In present study, for dimension $n$, the rhombus silicate and rhombus oxide networks are denoted by $(R H S L(n))$ and $(R H O X(n))$ respectively. 


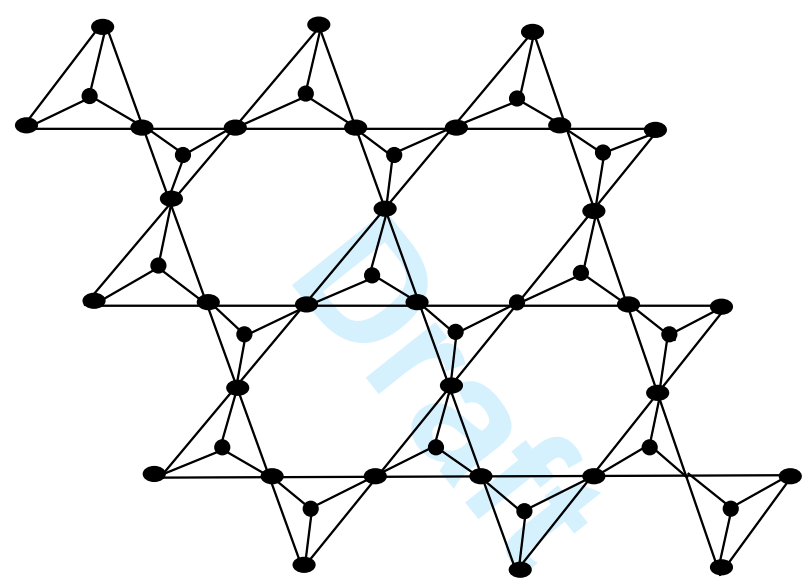

Figure 1: Rhombus silicate $R H S L(3)$ 


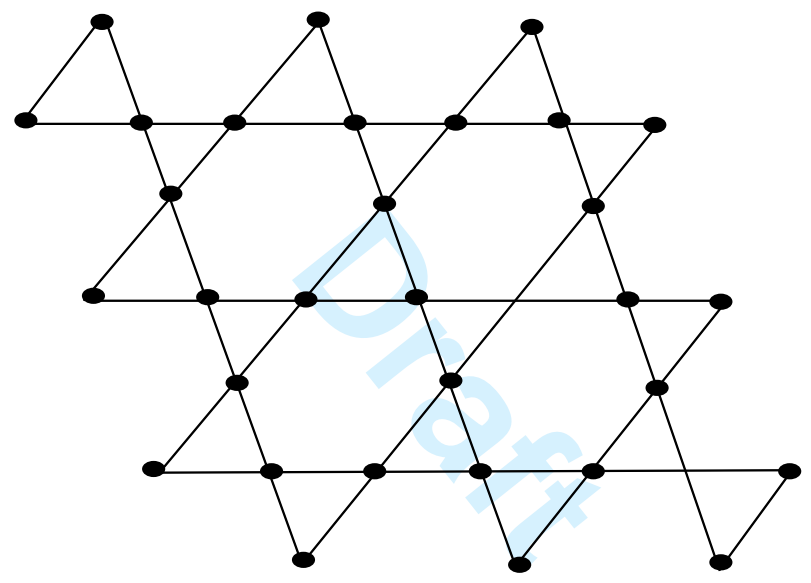

Figure 2: Rhombus oxide $R H O X(3)$ 


\section{Topological indices of the rhombus silicate network}

In this section, we compute the topological indices for rhombus silicate. In Theorem 3.1, Theorem 3.2 and Theorem 3.3, we find the topological indices which base on the degrees of the vertices such as general Randić, first general Zagreb, generalized Zagreb, multiplicative Zagreb, atom-bond connectivity (ABC), geometric-arithmetic (GA) and augmented Zagreb index (AZI). Moreover, Theorem 3.4 consists on the latest devolved topological indices which base on the sum of the degrees of neighbors of the vertices such as fourth version of $\mathrm{ABC}\left(A B C_{4}\right)$, the fifth version of $\mathrm{GA}\left(G A_{5}\right)$, and Sanskruti index (SI).

For this let us define the partitions of the vertex set $V(R H S L(n))$ and the edge set $E(R H S L(n))$ of $R H S L(n)$ with respect to degree of vertices. There are two types of vertices in $R H S L(n)$ namely of degree 3 and of degree 6 . Thus, we have

$$
V_{1}=\left\{v \in V(R H S L(n)) \mid d_{v}=3\right\} \text { and } V_{2}=\left\{v \in V(R H S L(n)) \mid d_{v}=6\right\}
$$

where $\left|V_{1}\right|=2 n(n+2)$ and $\left|V_{2}\right|=n(3 n-2)$. Consequently, $|V(R H S L(n))|=$ $v=\left|V_{1}\right|+\left|V_{2}\right|=5 n^{2}+2 n$. There are three types of edges with respect to degrees of end vertices in $R H S L(n)$ namely with degrees of end vertices 


\begin{tabular}{|l|l|l|l|}
\hline$E_{\{d(u), d(v)\}}$ & $E_{\{3,3\}}$ & $E_{\{3,6\}}$ & $E_{\{6,6\}}$ \\
\hline$\left|E_{\{d(u), d(v)\}}\right|$ & $4 n+2$ & $6 n^{2}+4 n-4$ & $6 n^{2}-8 n+2$ \\
\hline
\end{tabular}

Table 1: The edge partition of the edges of $R H S L(n)$ based on degrees of end vertices. 


\begin{tabular}{|l|l|}
\hline$E_{\left\{S_{u}, S_{v}\right\}}$ & $\left|E_{\left\{S_{u}, S_{v}\right\}}\right|$ \\
\hline$(12,12)$ & 6 \\
\hline$(12,24)$ & 6 \\
\hline$(15,24)$ & 8 \\
\hline$(18,24)$ & 2 \\
\hline$(24,27)$ & 8 \\
\hline$(15,15)$ & $4(n-1)$ \\
\hline$(18,27)$ & $4(2 n-3)$ \\
\hline$(27,27)$ & $8(n-2)+2$ \\
\hline$(15,27)$ & $8(2 n-3)$ \\
\hline$(27,30)$ & $8(n-2)$ \\
\hline$(18,30)$ & $2(n-2)(3 n-4)$ \\
\hline$(30,30)$ & $6(n-2)^{2}$ \\
\hline
\end{tabular}

Table 2: The edge partitions of $\operatorname{RHSL}(n)$ based on degree sum of the neighbors of end vertices. 
$\{3,3\}$, degrees of end vertices $\{3,6\}$ and degrees of end vertices $\{6,6\}$.

Thus, we have

$$
\begin{aligned}
& E_{\{3,3\}}=\left\{u v \in E(\operatorname{RHSL}(n)) \mid d_{u}=3, d_{v}=3\right\}, \\
& E_{\{3,6\}}=\left\{u v \in \operatorname{E}(\operatorname{RHSL}(n)) \mid d_{u}=3, d_{v}=6\right\},
\end{aligned}
$$

and

$$
E_{\{6,6\}}=\left\{u v \in E(R H S L(n)) \mid d_{u}=6, d_{v}=6\right\},
$$

where $\left|E_{\{3,3\}}\right|=4 n+2,\left|E_{\{3,6\}}\right|=6 n^{2}+4 n-4$ and $\left|E_{\{6,6\}}\right|=6 n^{2}-8 n+2$. Consequently, $|E(R H S L(n))|=e=\left|E_{1}\right|+\left|E_{2}\right|+\left|E_{3}\right|=12 n^{2}$. Similarly, we can define the partitions of the vertex set $V(R H S L(n))$ and the edge set $E(R H S L(n))$ of $R H S L(n)$ with respect to the sum of the degrees of neighbors of the vertices of $R H S L(n)$. Thus, for $u v \in E(R H S L(n))$, we obtain the Table 3.1 and Table 3.2 .

Theorem 3.1. Let $\Gamma \cong R H S L(n)$ be a rhombus silicate network with dimension $n$. Then, for $n \geq 2$, its Randić index $\left(R_{-\frac{1}{2}}(\Gamma)\right)$, reciprocal Randić index $\left(R_{\frac{1}{2}}(\Gamma)\right)$, second Zagreb index $\left(R_{1}(\Gamma)\right)$ and the general Randić index $R_{\alpha}(\Gamma)$ are given as 
$R_{\alpha}(\Gamma)=\left\{\begin{array}{l}\left(1+n^{2}\right)+\frac{6 n^{2}+4 n-4}{3 \sqrt{2}}, \text { for } \alpha=-\frac{1}{2}, \\ 36 n^{2}-36 n+3 \sqrt{2}\left(6 n^{2}+4 n-4\right)+18, \text { for } \alpha=\frac{1}{2}, \\ 324 n^{2}-180 n+18, \text { for } \alpha=1, \\ 6 n^{2}\left(18^{\alpha}+36^{\alpha}\right)+4 n\left(9^{\alpha}+18^{\alpha}-36^{\alpha} \times 2\right)+2\left(9^{\alpha}-2 \times 18^{\alpha}+36^{\alpha}\right), \\ \text { for } \alpha \in \mathbb{R} .\end{array}\right.$

Proof. Using Table 1 and the formulae defined in Section 2, we compute the required results as follows.

$$
\begin{aligned}
R_{-\frac{1}{2}}(\Gamma) & =\sum_{u v \in E(\Gamma)} \frac{1}{\sqrt{d_{u} d_{v}}} \\
& =\sum_{u v \in E_{\{3,3\}}} \frac{1}{\sqrt{d_{u} d_{v}}}+\sum_{u v \in E_{\{3,6\}}} \frac{1}{\sqrt{d_{u} d_{v}}}+\sum_{u v \in E_{\{6,6\}}} \frac{1}{\sqrt{d_{u} d_{v}}} \\
= & \frac{1}{\sqrt{9}}(4 n+2)+\left(6 n^{2}+4 n-4\right) \frac{1}{\sqrt{18}}+\left(6 n^{2}-8 n+2\right) \frac{1}{\sqrt{36}} \\
= & \frac{4 n+2}{3}+\frac{\left(6 n^{2}+4 n-4\right)}{3 \sqrt{2}}+\frac{\left(6 n^{2}-8 n+2\right)}{6} \\
= & \frac{8 n+4+6 n^{2}-8 n+2}{+} \frac{6 n^{2}+4 n-4}{3 \sqrt{2}} \\
= & \left(1+n^{2}\right)+\frac{6 n^{2}+4 n-4}{3 \sqrt{2}} .
\end{aligned}
$$




$$
\begin{aligned}
R_{\frac{1}{2}}(\Gamma)= & \sum_{u v \in E(\Gamma)} \sqrt{d_{u} d_{v}} \\
& =\sum_{u v \in E_{\{3,3\}}} \sqrt{d_{u} d_{v}}+\sum_{u v \in E_{\{3,6\}}} \sqrt{d_{u} d_{v}}+\sum_{u v \in E_{\{6,6\}}} \sqrt{d_{u} d_{v}} \\
& =(4 n+2) \sqrt{9}+\left(6 n^{2}+4 n-4\right) \sqrt{18}+\left(6 n^{2}-8 n+2\right) \sqrt{36} \\
& =12 n+6+3 \sqrt{2}\left(6 n^{2}+4 n-4\right)+36 n^{2}-48 n+12 \\
& =36 n^{2}-36 n+3 \sqrt{2}\left(6 n^{2}+4 n-4\right)+18 .
\end{aligned}
$$

$$
\begin{aligned}
R_{1}(\Gamma)= & \sum_{u v \in E(T)}\left(d_{v} d_{u}\right) \\
& =\sum_{u v \in E_{\{3,3\}}}\left(d_{v} d_{u}\right)+\sum_{u v \in E_{\{3,6\}}}\left(d_{v} d_{u}\right)+\sum_{u v \in E_{\{6,6\}}}\left(d_{v} d_{u}\right) \\
& =(4 n+2)(9)+\left(6 n^{2}+4 n-4\right)(18)+\left(6 n^{2}-8 n+2\right)(36) \\
& =36 n+18+108 n^{2}+72 n-72+216 n^{2}-288 n+72 \\
& =324 n^{2}-180 n+18 .
\end{aligned}
$$

$$
\begin{aligned}
R_{\alpha}(\Gamma)= & \sum_{u v \in E(\Gamma)}\left(d_{u} d_{v}\right)^{\alpha} \\
& =\sum_{u v \in E_{3,3}}\left(d_{u} d_{v}\right)^{\alpha}+\sum_{u v \in E_{3,6}}\left(d_{u} d_{v}\right)^{\alpha}+\sum_{u v \in E_{6,6}}\left(d_{u} d_{v}\right)^{\alpha} \\
& =(4 n+2)(9)^{\alpha}+\left(6 n^{2}+4 n-4\right)(18)^{\alpha}+\left(6 n^{2}-8 n+2\right)(36) \\
& =4 n \times 9^{\alpha}+\left(6 n^{2}+4 n-4\right)(18)^{\alpha}+\left(6 n^{2}-8 n+2\right)(36)^{\alpha} \\
& =6 n^{2}\left(18^{\alpha}+36^{\alpha}\right)+4 n\left(9^{\alpha}+18^{\alpha}-36^{\alpha} \times 2\right)+2\left(9^{\alpha}-2 \times 18^{\alpha}+36^{\alpha}\right) .
\end{aligned}
$$

Theorem 3.2. Let $\Gamma \cong R H S L(n)$ be a rhombus silicate network with 
dimension $n$. Then, for $n \geq 2$, first general Zagreb $\left(M_{1}^{\alpha}(\Gamma)\right)$, generalized Zagreb $\left(M_{r, s}(\Gamma)\right)$, first multiplicative Zagreb $\left(P M_{1}(\Gamma)\right)$ and the second multiplicative Zagreb $\left(P M_{2}(\Gamma)\right)$ indices are given by

(i) $M_{1}^{\alpha}(\Gamma)=n^{2}\left(2 \times 3^{\alpha}+3 \times 6^{\alpha}\right)+2 n\left(2 \times 3^{\alpha}-6^{\alpha}\right)$,

(ii) $M_{r, s}(\Gamma)=\left(2 \times 3^{r+s}\right)\left[3 n^{2}\left(2^{r}+2^{s}+2\left(2^{r} 2^{s}\right)\right)+2 n\left(2+2^{r}+2^{s}-2^{2}\left(2^{r}\right)\left(2^{s}\right)+\right.\right.$ $2\left(1-2^{r}-2^{s}+\left(2^{r}\right)\left(2^{s}\right)\right]$,

(iii) $P M_{1}(\Gamma)=5184(1+2 n)\left(1-4 n+3 n^{2}\right)\left(-2+2 n+3 n^{2}\right)$,

(iv) $P M_{2}(\Gamma)=46656(1+2 n)\left(1-4 n+3 n^{2}\right)\left(-2+2 n+3 n^{2}\right)$.

Proof. Using Table 1 and the formulae defined in Section 2, we compute the required results as follows.

(i)

$$
\begin{aligned}
M_{1}^{\alpha}(\Gamma)= & \sum_{u \in V(\Gamma)}\left(d_{u}\right)^{\alpha} \\
& =\left(2 n^{2}+4 n\right)(3)^{\alpha}+\left(3 n^{2}-2 n\right)(6)^{\alpha} \\
& =n^{2}\left(2 \times 3^{\alpha}+3 \times 6^{\alpha}\right)+2 n\left(2 \times 3^{\alpha}-6^{\alpha}\right),
\end{aligned}
$$


(ii)

$$
\begin{aligned}
M_{r, s}(T)= & \sum_{u v \in E(T)}\left(d_{u}^{r} d_{v}^{s}+d_{v}^{r} d_{u}^{s}\right) \\
& =\sum_{u v \in E_{\{3,3\}}}\left(d_{u}^{r} d_{v}^{s}+d_{v}^{r} d_{u}^{s}\right)+\sum_{u v \in E_{\{3,6\}}}\left(d_{u}^{r} d_{v}^{s}+d_{v}^{r} d_{u}^{s}\right) \\
& +\sum_{u v \in E_{\{6,6\}}}\left(d_{u}^{r} d_{v}^{s}+d_{v}^{r} d_{u}^{s}\right) \\
& =(4 n+2)\left(3^{r} 3^{s}+3^{s} 3^{r}\right)+\left(6 n^{2}+4 n-4\right)\left(3^{r} 6^{s}+6^{r} 3^{s}\right. \\
& +\left(6 n^{2}-8 n+2\right)\left(6^{r} 6^{s}+6^{s} 6^{r}\right) \\
& =(4 n+2)\left(2 \times 3^{r+s}\right)+2\left(3 n^{2}+2 n-2\right)\left(3^{r+s}\right)\left(2^{r}+2^{s}\right) \\
& +2\left(3 n^{2}-4 n+1\right)\left((2)\left(2^{r}\right)\left(2^{s}\right)\right)\left(3^{r+s}\right) \\
& =\left(2 \times 3^{r+s}\right)\left[(4 n+2)+\left(3 n^{2}+2 n-2\right)\left(2^{r}+2^{s}\right)\right. \\
& +\left(3 n^{2}-4 n+1\right)\left((2)\left(2^{r}\right)\left(2^{s}\right)\right) \\
& =\left(2 \times 3^{r+s}\right)\left[3 n^{2}\left(2^{r}+2^{s}+2\left(2^{r}\right)\left(2^{s}\right)\right)+2 n\left(2+2^{r}+2^{s}\right.\right. \\
& \left.-\left(2^{2}\right)\left(2^{r}\right)\left(2^{s}\right)\right)+2\left(1-2^{r}-2^{s}+\left(2^{r}\right)\left(2^{s}\right)\right],
\end{aligned}
$$

(iii)

$$
\begin{aligned}
P M_{1}(\Gamma)= & \prod_{u v \in E(T)}\left(d_{v}+d_{u}\right) \\
& =\prod_{u v \in E_{\{3,3\}}}\left(d_{v}+d_{u}\right) \times \prod_{u v \in E_{\{3,6\}}}\left(d_{v}+d_{u}\right) \times \prod_{u v \in E_{\{6,6\}}}\left(d_{v}+d_{u}\right) \\
& =(4 n+2)(6)\left(6 n^{2}+4 n-4\right)(9)\left(6 n^{2}-8 n+2\right)(12) \\
& =(4 n+2)\left(54 n^{2}+36 n-36\right)\left(72 n^{2}-96 n+24\right) \\
& =5184(1+2 n)\left(1-4 n+3 n^{2}\right)\left(-2+2 n+3 n^{2}\right),
\end{aligned}
$$


(iv)

$$
\begin{aligned}
P M_{2}(\Gamma)= & \prod_{u v \in E(T)}\left(d_{v} \times d_{u}\right) \\
& =\prod_{u v \in E_{\{3,3\}}}\left(d_{v} d_{u}\right) \times \prod_{u v \in E_{\{3,6\}}}\left(d_{v} d_{u}\right) \times \prod_{u v \in E_{\{6,6\}}}\left(d_{v} d_{u}\right) \\
& =9(4 n+2)\left(6 n^{2}+4 n-4\right)(18)\left(6 n^{2}-8 n+2\right)(36) \\
& =(36 n+18)\left(108 n^{2}+72 n-72\right)\left(216 n^{2}-288 n+72\right) \\
& =46656(1+2 n)\left(1-4 n+3 n^{2}\right)\left(-2+2 n+3 n^{2}\right) .
\end{aligned}
$$

Theorem 3.3. Let $\Gamma \cong R H S L(n)$ be a rhombus silicate network with dimension $n$. Then, for $n \geq 2$, atom-bond connectivity $(A B C(\Gamma))$, geometricarithmetic $(G A(\Gamma))$ and augmented Zagreb index (AZI) are given by

(i) $A B C(\Gamma)=6.9039 n^{2}+0.9447 n-0.1070$,

(ii) $G A(\Gamma)=11.6568 n^{2}-0.2287 n+0.2287$,

(iii) $A Z I(\Gamma)=381.9534 n^{2}-259.674 n+48.0816$.

Proof. Using Table 1 and the formulae defined in Section 2, we compute the required results as follows.

(i)

$$
\begin{aligned}
A B C(\Gamma)= & \sum_{u v \in E(\Gamma)} \sqrt{\frac{d_{u}+d_{v}-2}{d_{u} \times d_{v}}}=\sum_{u v \in E_{\{3,3\}}} \sqrt{\frac{d_{u}+d_{v}-2}{d_{u} \times d_{v}}} \\
& +\sum_{u v \in E_{\{3,6\}}} \sqrt{\frac{d_{u}+d_{v}-2}{d_{u} \times d_{v}}}+\sum_{u v \in E_{\{6,6\}}} \sqrt{\frac{d_{u}+d_{v}-2}{d_{u} \times d_{v}}} \\
& =(4 n+2) \sqrt{\frac{3+3-2}{3 \times 3}}+\left(6 n^{2}+4 n-4\right) \sqrt{\frac{3+6-2}{3 \times 6}}
\end{aligned}
$$




$$
\begin{aligned}
& +\left(6 n^{2}-8 n+2\right) \sqrt{\frac{6+6-2}{6 \times 6}} \\
& =\frac{2}{3}(4 n+2)+\left(6 n^{2}+4 n-4\right) \frac{\sqrt{7}}{3 \sqrt{2}}+\left(6 n^{2}-8 n+2\right) \frac{\sqrt{10}}{6} \\
& =\frac{2}{3}(4 n+2)+\left(3 n^{2}+2 n-2\right) \frac{\sqrt{14}}{3}+\left(3 n^{2}-4 n+1\right) \frac{\sqrt{10}}{3} \\
& =\frac{1}{3}\left[2(4 n+2)+\sqrt{14}\left(3 n^{2}+2 n-2\right)+\sqrt{10}\left(3 n^{2}-4 n+1\right)\right] \\
& =6.9039 n^{2}+0.9447 n-0.1070 .
\end{aligned}
$$

(ii)

$$
\begin{aligned}
G A(\Gamma)= & \sum_{u v \in E(\Gamma)} \frac{2 \sqrt{d_{u} \times d_{v}}}{d_{u}+d_{v}}=\sum_{u v \in E\{3,3\}} \frac{2 \sqrt{d_{u} \times d_{v}}}{d_{u}+d_{v}} \\
& +\sum_{u v \in E\{3,6\}} \frac{2 \sqrt{d_{u} \times d_{v}}}{d_{u}+d_{v}}+\sum_{u v \in E\{6,6\}} \frac{2 \sqrt{d_{u} \times d_{v}}}{d_{u}+d_{v}} \\
& =\frac{2 \times(4 n+2) \sqrt{3 \times 3}}{3+3}+\frac{2\left(6 n^{2}+4 n-4\right) \sqrt{3 \times 6}}{3+6} \\
& +\frac{\left(6 n^{2}-8 n+2\right) \times 2 \sqrt{6 \times 6}}{6+6} \\
& =(4 n+2)+\frac{2 \times 3 \sqrt{2}}{9}\left(6 n^{2}+4 n-4\right)+\left(6 n^{2}-8 n+2\right) \\
& =\left(6 n^{2}-4 n+4\right)+\frac{2 \sqrt{2}}{3}\left(6 n^{2}+4 n-4\right) \\
& =11.6568 n^{2}-0.2287 n+0.2287 .
\end{aligned}
$$


(iii)

$$
\begin{aligned}
A Z I(T)= & \sum_{u v \in E(T)}\left(\frac{d_{u} \times d_{v}}{d_{u}+d_{v}-2}\right)^{3}=\sum_{u v \in E_{\{3,3\}}}\left(\frac{d_{u} \times d_{v}}{d_{u}+d_{v}-2}\right)^{3} \\
& +\sum_{u v \in E_{\{3,6\}}}\left(\frac{d_{u} \times d_{v}}{d_{u}+d_{v}-2}\right)^{3}+\sum_{u v \in E_{\{6,6\}}}\left(\frac{d_{u} \times d_{v}}{d_{u}+d_{v}-2}\right)^{3} \\
& =(4 n+2)\left(\frac{3 \times 3}{3+3-2}\right)^{3}+\left(6 n^{2}+4 n-4\right)\left(\frac{3 \times 6}{3+6-2}\right)^{3} \\
& \quad+\left(6 n^{2}-8 n+2\right)\left(\frac{6 \times 6}{6+6-2}\right)^{3} \\
= & (4 n+2)(11.3906)+\left(6 n^{2}+4 n-4\right)(17.0029) \\
+ & \left(6 n^{2}-8 n+2\right)(46.656) \\
= & 45.5624 n+22.7812+102.0174 n^{2}+68.0116 n-68.0116 \\
+ & 279.936 n^{2}-373.248 n+93.312 \\
= & 381.9534 n^{2}-259.674 n+48.0816 .
\end{aligned}
$$

Theorem 3.4. Let $\Gamma \cong R H S L(n)$ be a rhombus silicate network with dimension $n$. Then, for $n \geq 2$, the fourth version of $\mathrm{ABC}\left(A B C_{4}\right)$, the fifth version of GA $\left(G A_{5}\right)$, and Sanskruti indices are given as

(i) $A B C_{4}(\Gamma)=3.2743 n^{2}+1.1107 n+0.1107$,

(ii) $G A_{5}(\Gamma)=11.8095 n^{2}-0.2047 n+0.1636$,

(iii) $S(\Gamma)=32124 n^{2}-44196.4 n+15280.4$

Proof. Using Table 2 and the formulae defined in Section 2, we compute the required results as follow. 
(i)

$$
\begin{aligned}
A B C_{4}= & \sum_{u v \in E(\Gamma)} \sqrt{\frac{S_{u}+S_{v}-2}{S_{u} \times S_{v}}}=6 \sqrt{\frac{12+12-2}{12 \times 12}} \\
& +6 \sqrt{\frac{12+24-2}{12 \times 24}}+8 \sqrt{\frac{15+24-2}{15 \times 24}}+2 \sqrt{\frac{18+24-2}{18 \times 24}} \\
& +8 \sqrt{\frac{24+27-2}{24 \times 27}}+4(n-1) \sqrt{\frac{15+15-2}{15 \times 15}} \\
& +4(2 n-3) \sqrt{\frac{18+27-2}{18 \times 27}}+[8(n-2)+2] \sqrt{\frac{27+27-2}{27 \times 27}} \\
& +8(2 n-3) \sqrt{\frac{15+27-2}{15 \times 27}}+8(n-2) \sqrt{\frac{27+30-2}{27 \times 30}} \\
& +2(n-2)(3 n-4) \sqrt{\frac{18+30-2}{18 \times 30}}+6(n-2)^{2} \sqrt{\frac{30+30-2}{30 \times 30}} \\
& =2.3452+2.0616+2.5647+0.6086+2.1999+1.4111(n-1) \\
& +1.1898(2 n-3)+2.1368(n-2)+0.5342+2.5142(2 n-3) \\
& +2.0846(n-2)+0.5837(n-2)(3 n-4)+1.5232(n-2)^{2} \\
& =10.3142+1.4111(n-1)+1.1898(2 n-3) \\
& +2.1368(n-2)+2.5142(2 n-3)+2.0846(n-2) \\
& +0.5837(n-2)(3 n-4)+1.5232(n-2)^{2} \\
& =3.2743 n^{2}+1.1107 n+0.1107
\end{aligned}
$$


(ii)

$$
\begin{aligned}
G A_{5}(\Gamma)= & \sum_{u v \in E(\Gamma)} \frac{2 \sqrt{S_{u} \times S_{v}}}{S_{u}+S_{v}} \\
& =\frac{12 \sqrt{12 \times 12}}{12+12}+\frac{12 \sqrt{12 \times 24}}{12+24}+\frac{16 \sqrt{15 \times 24}}{15+24} \\
& +\frac{4 \sqrt{18 \times 24}}{18+24}+\frac{16 \sqrt{24 \times 27}}{24+27}+\frac{8(n-1) \sqrt{15 \times 15}}{15+15} \\
& +\frac{8(2 n-3) \sqrt{18 \times 27}}{18+27}+\frac{[16(n-2)+4] \sqrt{27 \times 27}}{27+27} \\
& +\frac{16(2 n-3) \sqrt{15 \times 27}}{15+27}+\frac{16(n-2) \sqrt{27 \times 30}}{27+30}+ \\
& \frac{4(n-2)(3 n-4) \sqrt{18 \times 30}}{18+30}+\frac{12(n-2)^{2} \sqrt{30 \times 30}}{30+30} \\
& =6+5.6569+7.7841+1.9794+7.9861+4(n-1) \\
& +3.9192(2 n-3)+8(n-2)+2+7.6665(2 n-3) \\
& +7.9889(n-2)+1.9365(n-2)(3 n-4)+6(n-2)^{2} \\
& =11.8095 n^{2}-0.2047 n+0.1636 .
\end{aligned}
$$

(iii)

$$
\begin{aligned}
S(\Gamma)= & \sum_{u v \in E(\Gamma)}\left(\frac{S_{u} \times S_{v}}{S_{u}+S_{v}-2}\right)^{3} \\
& =6\left(\frac{12 \times 12}{12+12-2}\right)^{3}+6\left(\frac{12 \times 24}{12+24-2}\right)^{3}+8\left(\frac{15 \times 24}{15+24-2}\right)^{3} \\
& +2\left(\frac{18 \times 24}{18+24-2}\right)^{3}+8\left(\frac{24 \times 27}{24+27-2}\right)^{3}+4(n-1)\left(\frac{15 \times 15}{15+15-2}\right)^{3} \\
& +4(2 n-3)\left(\frac{18 \times 27}{18+27-2}\right)^{3}+[8(n-2)+2]\left(\frac{27 \times 27}{27+27-2}\right)^{3} \\
& +8(2 n-3)\left(\frac{15 \times 27}{15+27-2}\right)^{3}+8(n-2)\left(\frac{27 \times 30}{27+30-2}\right)^{3} \\
& +2(n-2)(3 n-4)\left(\frac{18 \times 30}{18+30-2}\right)^{3}+6(n-2)^{2}\left(\frac{30 \times 30}{30+30-2}\right)^{3}
\end{aligned}
$$




$$
\begin{aligned}
& =1682.5605+3646.6322+7368.7245+2519.424+18502.3446 \\
& +2075.5512(n-1)+5775.1522(2 n-3)+22042.5859(n-2)+5510.6464 \\
& +8303.7656(2 n-3)+25553.8873(n-2) \\
& +3235.4730(n-2)(3 n-4)+22417.8933(n-2)^{2} \\
& =39230.3320+2075.5512(n-1)+5775.1522(2 n-3)+22042.5859(n-2) \\
& +8303.7656(2 n-3)+25553.8873(n-2)+3235.4730(n-2)(3 n-4) \\
& +22417.8933(n-2)^{2} \\
& =32124 n^{2}-44196.4 n+15280.4 .
\end{aligned}
$$

\section{Topological indices of the rhombus oxide network}

In this section, we compute the topological indices such as general Randić, first general Zagreb, generalized Zagreb, multiplicative Zagreb, atom-bond connectivity (ABC), geometric-arithmetic (GA), augmented Zagreb, fourth version of $\mathrm{ABC}\left(A B C_{4}\right)$, the fifth version of GA $\left(G A_{5}\right)$, and Sanskruti index (SI) for the rhombus oxide $(R H O X(n))$ network.

Before to the main theorems, we define the partitions of the vertex set $V(R H O X(n))$ and the edge set $E(R H O X(n))$ of $R H O X(n)$ with respect to degree of vertices. We note that each vertex $\operatorname{RHOX}(n)$ is of degree 2 


\begin{tabular}{|l|l|l|l|}
\hline$E_{\{d(u), d(v)\}}$ & $E_{\{2,2\}}$ & $E_{\{2,4\}}$ & $E_{\{4,4\}}$ \\
\hline$\left|E_{\{d(u), d(v)\}}\right|$ & 2 & $4(2 n-1)$ & $6 n^{2}-8 n+2$ \\
\hline
\end{tabular}

Table 3: The edge partition based on the degrees of the end vertices of $\operatorname{RHOX}(n)$. 


\begin{tabular}{|l|l|}
\hline$E_{\left\{S_{u}, S_{v}\right\}}$ & $\left|E_{\left\{S_{u}, S_{v}\right\}}\right|$ \\
\hline$(6,6)$ & 2 \\
\hline$(6,12)$ & 4 \\
\hline$(8,12)$ & 4 \\
\hline$(8,14)$ & $4(2 n-3)$ \\
\hline$(12,14)$ & 8 \\
\hline$(14,14)$ & $2(4 n-7)$ \\
\hline$(14,16)$ & $8(n-2)$ \\
\hline$(16,16)$ & $6(n-2)^{2}$ \\
\hline
\end{tabular}

Table 4: The edge partition based on the degree sum of neighbors of the end vertices of $R H O X(n)$. 
or of degree 4. Thus, we have

$V_{1}=\left\{v \in V(R H O X(n)) \mid d_{v}=2\right\}$ and $V_{2}=\left\{v \in V(\operatorname{RHOX}(n)) \mid d_{v}=4\right\}$,

where $\left|V_{1}\right|=4 n$ and $\left|V_{2}\right|=3 n^{2}-2 n$. Consequently, $|V(R H O X(n))|=$ $v=\left|V_{1}\right|+\left|V_{2}\right|=3 n^{2}+2 n$. Moreover, there are three types of edges with respect to degrees of end vertices in $\operatorname{RHOX}(n)$ namely with degrees of end vertices $\{2,2\}$, degrees of end vertices $\{2,4\}$ and degrees of end vertices $\{4,4\}$. Thus, we have

$$
\begin{aligned}
& E_{\{2,2\}}=\left\{u v \in E(\operatorname{RHOX}(n)) \mid d_{u}=2, d_{v}=2\right\}, \\
& E_{\{2,4\}}=\left\{u v \in E(\operatorname{RHOX}(n)) \mid d_{u}=2, d_{v}=4\right\}, \\
& E_{\{4,4\}}=\left\{u v \in E(\operatorname{RHOX}(n)) \mid d_{u}=4, d_{v}=4\right\},
\end{aligned}
$$

and

where $\left|E_{\{2,2\}}\right|=2,\left|E_{\{2,4\}}\right|=4(2 n-1)$ and $\left|E_{\{4,4\}}\right|=6 n^{2}-8 n+2$. Consequently, $|E(\operatorname{RHOX}(n))|=e=\left|E_{\{2,2\}}\right|+\left|E_{\{2,4\}}\right|+\left|E_{\{4,4\}}\right|=6 n^{2}$. Similarly, we can define the partitions of the vertex set $V(R H O X(n))$ and the edge set $E(R H O X(n))$ of $R H O X(n)$ with respect to the sum of the degrees of neighbors of the vertices of $\operatorname{RHOX}(n)$. Thus, for $u v \in E(R H O X(n))$, we have the Table 4.1 and Table 4.2 .

Theorem 4.1. Let $\Gamma \cong R H O X(n)$ be a rhombus oxide network with dimension $n$. Then, for $n \geq 2$, its Randić index $\left(R_{-\frac{1}{2}}(\Gamma)\right)$, reciprocal Randić index $\left(R_{\frac{1}{2}}(\Gamma)\right)$, second Zagreb index $\left(R_{1}(\Gamma)\right)$ and the general Randić index $R_{\alpha}(\Gamma)$ are given as 
$R_{\alpha}(\Gamma)=\left\{\begin{array}{l}\frac{1}{2}\left(3 n^{2}-4 n+3+2 \sqrt{2}(2 n-1)\right), \text { for } \alpha=-\frac{1}{2}, \\ 24 n^{2}-32 n+8 \sqrt{2}(2 n-1)+12, \text { for } \alpha=\frac{1}{2}, \\ 96 n^{2}-64 n+8, \text { for } \alpha=1, \\ n^{2}\left(6 \times 16^{\alpha}\right)+8 n\left(8^{\alpha}-16^{\alpha}\right)+\left(2 \times 4^{\alpha}\right)\left[1+2^{\alpha}\left(2^{\alpha}-2\right)\right], \\ \text { for } \alpha \in \mathbb{R} .\end{array}\right.$

Proof. Using Table 3 and the formulae defined in Section 2, we compute the required results as follow.

$$
\begin{aligned}
R_{-\frac{1}{2}}(\Gamma)= & \sum_{u v \in E(\Gamma)} \frac{1}{\sqrt{d_{u} \times d_{v}}} \\
& =\sum_{u v \in E_{\{2,2\}}} \frac{1}{\sqrt{d_{u} \times d_{v}}}+\sum_{u v \in E_{\{2,4\}}} \frac{1}{\sqrt{d_{u} \times d_{v}}}+\sum_{u v \in E_{\{4,4\}}} \frac{1}{\sqrt{d_{u} \times d_{v}}} \\
& =\frac{2}{\sqrt{2 \times 2}}+\frac{4(2 n-1)}{\sqrt{2 \times 4}}+\operatorname{frac}\left(6 n^{2}-8 n+2\right) \sqrt{4 \times 4} \\
& =\frac{2}{2}+\frac{4(2 n-1)}{\sqrt{2 \times 4}}+\frac{\left(6 n^{2}-8 n+2\right)}{\sqrt{4 \times 4}} \\
& =\frac{2}{2}+\frac{6 n^{2}-8 n+2}{4}+\frac{4(2 n-1)}{2 \sqrt{2}} \\
& =\frac{4+6 n^{2}-8 n+2}{4}+\frac{4(2 n-1)}{2 \sqrt{2}} \\
& =\frac{6 n^{2}-8 n+2}{4}+\sqrt{2}(2 n-1) \\
& =\frac{1}{2}\left(3 n^{2}-4 n+3+2 \sqrt{2}(2 n-1)\right),
\end{aligned}
$$




$$
\begin{aligned}
R_{\frac{1}{2}}(\Gamma)= & \sum_{u v \in E(\Gamma)} \sqrt{d_{u} \times d_{v}} \\
& =\sum_{u v \in E_{\{2,2\}}} \sqrt{d_{u} \times d_{v}}+\sum_{u v \in E_{\{2,4\}}} \sqrt{d_{u} \times d_{v}}+\sum_{u v \in E_{\{4,4\}}} \sqrt{d_{u} \times d_{v}} \\
& =2 \sqrt{2 \times 2}+4(2 n-1) \sqrt{2 \times 4}+\left(6 n^{2}-8 n+2\right) \sqrt{4 \times 4} \\
& =4+8 \sqrt{2}(2 n-1)+4\left(6 n^{2}-8 n+2\right) \\
& =4+8 \sqrt{2}(2 n-1)+24 n^{2}-32 n+8 \\
& =24 n^{2}-32 n+8 \sqrt{2}(2 n-1)+12,
\end{aligned}
$$

$$
\begin{aligned}
R_{1}(\Gamma)= & \sum_{u v \in E(T)}\left(d_{v} \times d_{u}\right) \\
& =\sum_{u v \in E_{\{2,2\}}}\left(d_{v} \times d_{u}\right)+\sum_{u v \in E_{\{2,4\}}}\left(d_{v} \times d_{u}\right)+\sum_{u v \in E_{\{4,4\}}}\left(d_{v} \times d_{u}\right) \\
& =2(2 \times 2)+4(2 n-1)(2 \times 4)+\left(6 n^{2}-8 n+2\right)(4 \times 4) \\
& =8+32(2 n-1)+16\left(6 n^{2}-8 n+2\right) \\
& =96 n^{2}-64 n+8,
\end{aligned}
$$




$$
\begin{aligned}
R_{\alpha}(\Gamma)= & \sum_{u v \in E(\Gamma)}\left(d_{u} \times d_{v}\right)^{\alpha} \\
& =\sum_{u v \in E_{2,2}}\left(d_{u} d_{v}\right)^{\alpha}+\sum_{u v \in E_{2,4}}\left(d_{u} d_{v}\right)^{\alpha}+\sum_{u v \in E_{4,4}}\left(d_{u} d_{v}\right)^{\alpha} \\
& =2(2 \times 2)^{\alpha}+4(2 n-1)(2 \times 4)^{\alpha}+\left(6 n^{2}-8 n+2\right)(4 \times 4) \\
& =2 \times 4^{\alpha}+4(2 n-1)(8)^{\alpha}+\left(6 n^{2}-8 n+2\right)(16)^{\alpha} \\
& =\left(6 n^{2} \times 16^{\alpha}\right)+\left(8 n \times 8^{\alpha}\right)-\left(8 n \times 16^{\alpha}\right)+\left(2 \times 4^{\alpha}\right)+\left(2 \times 16^{\alpha}\right) \\
& -\left(4 \times 8^{\alpha}\right) \\
& =n^{2}\left(6 \times 16^{\alpha}\right)+8 n\left(8^{\alpha}-16^{\alpha}\right)+\left(2 \times 4^{\alpha}\right)\left[1+2^{\alpha}\left(2^{\alpha}-2\right)\right] .
\end{aligned}
$$

Theorem 4.2. Let $\Gamma \cong R H O X(n)$ be a rhombus oxide with dimension $n$. Then, for $n \geq 2$, first general Zagreb $\left(M_{1}^{\alpha}(\Gamma)\right)$, generalized Zagreb $\left(M_{r, s}(\Gamma)\right)$, first multiplicative Zagreb $\left(P M_{1}(\Gamma)\right)$ and the second multiplicative Zagreb $\left(P M_{2}(\Gamma)\right)$ indices are given by

(i) $M_{1}^{\alpha}(\Gamma)=2^{\alpha} \times 4 n+4^{\alpha}\left(3 n^{2}-2 n\right)$,

(ii) $M_{r, s}(T)=2^{r+s+2}\left[3 n^{2}\left(2^{r+s}\right)+2 n\left(2^{r}+2^{s}-2^{r+s+1}\right)+\left(1-2^{r}-2^{s}+2^{r+s+1}\right)\right]$,

(iii) $P M_{1}(\Gamma)=18432 n^{3}-33792 n^{2}+18432 n-3072$,

(iv) $P M_{2}(\Gamma)=49152 n^{3}-90112 n^{2}+49152 n-8192$.

Proof. Using Table 3 and the formulae defined in Section 2, we compute the required results as follows. (i)

$$
\begin{aligned}
M_{1}^{\alpha}(\Gamma)= & \sum_{u \in V(\Gamma)}\left(d_{u}\right)^{\alpha} \\
& =4 n(2)^{\alpha}+\left(3 n^{2}-2 n\right)(4)^{\alpha} \\
& =2^{\alpha} \times 4 n+4^{\alpha}\left(3 n^{2}-2 n\right) .
\end{aligned}
$$


(ii)

$$
\begin{aligned}
M_{r, s}(T)= & \sum_{u v \in E(T)}\left(d_{u}^{r} d_{v}^{s}+d_{v}^{r} d_{u}^{s}\right)=\sum_{u v \in E_{\{2,2\}}}\left(d_{u}^{r} d_{v}^{s}+d_{v}^{r} d_{u}^{s}\right) \\
& +\sum_{u v \in E_{\{2,4\}}}\left(d_{u}^{r} d_{v}^{s}+d_{v}^{r} d_{u}^{s}\right)+\sum_{u v \in E_{\{4,4\}}}\left(d_{u}^{r} d_{v}^{s}+d_{v}^{r} d_{u}^{s}\right) \\
& =2\left(2^{r} \times 2^{s}+2^{s} \times 2^{r}\right)+4(2 n-1)\left(2^{r} \times 4^{s}+2^{s} \times 4^{r}\right) \\
& +\left(6 n^{2}-8 n+2\right)\left(4^{r} \times 4^{s}+4^{s} \times 4^{r}\right) \\
& =\left(4 \times 2^{r+s}\right)\left[1+(2 n-1)\left(2^{r}+2^{s}\right)+\left(3 n^{2}-4 n+1\right) 2^{r+s}\right] \\
& =2^{r+s+2}\left[3 n^{2}\left(2^{r+s}\right)+2 n\left(2^{r}+2^{s}-2^{r+s+1}\right)\right. \\
& \left.+\left(1-2^{r}-2^{s}+2^{r+s+1}\right)\right]
\end{aligned}
$$

(iii)

$$
\begin{aligned}
P M_{1}(\Gamma)= & \prod_{u v \in E(T)}\left(d_{v}+d_{u}\right) \\
& =\prod_{u v \in E_{\{2,2\}}}\left(d_{v}+d_{u}\right) \times \prod_{u v \in E_{\{2,4\}}}\left(d_{v}+d_{u}\right) \times \prod_{u v \in E_{\{4,4\}}}\left(d_{v}+d_{u}\right) \\
& =2(2+2) \times 4(2 n-1)(2+4) \times\left(6 n^{2}-8 n+2\right)(4+4) \\
& =8 \times 24(2 n-1) \times 8\left(6 n^{2}-8 n+2\right) \\
& =18432 n^{3}-33792 n^{2}+18432 n-3072,
\end{aligned}
$$


(iv)

$$
\begin{aligned}
P M_{2}(\Gamma)= & \prod_{u v \in E(T)}\left(d_{v} \times d_{u}\right) \\
& =\prod_{u v \in E_{\{2,2\}}}\left(d_{v} \times d_{u}\right) \times \prod_{u v \in E_{\{2,4\}}}\left(d_{v} \times d_{u}\right) \times \prod_{u v \in E_{\{4,4\}}}\left(d_{v} \times d_{u}\right) \\
& =2(2 \times 2) \times 4(2 n-1)(2 \times 4) \times\left(6 n^{2}-8 n+2\right)(4 \times 4) \\
& =4096\left(12 n^{3}-22 n^{2}+4 n-2\right) \\
& =49152 n^{3}-90112 n^{2}+49152 n-8192 .
\end{aligned}
$$

Theorem 4.3. Let $\Gamma \cong \operatorname{RHOX}(n)$ be a rhombus oxide network with dimension $n$. Then, for $n \geq 2$, atom-bond connectivity $(A B C(\Gamma))$ index, geometric-arithmetic $(G A(\Gamma))$ index and augmented Zagreb index (AZI) are given by

(i) $A B C(\Gamma)=3.6742 n^{2}+0.7578 n-0.1894$,

(ii) $G A(\Gamma)=6 n^{2}-0.4575 n+0.2287$,

(iii) $A Z I(\Gamma)=113.7774 n^{2}-87.7032 n+21.9258$.

Proof. Using Table 3 and the formulae defined in Section 2, we compute the required results as follows. 
(i)

$$
\begin{aligned}
& A B C(\Gamma)=\sum_{u v \in E(\Gamma)} \sqrt{\frac{d_{u}+d_{v}-2}{d_{u} \times d_{v}}}=\sum_{u v \in E_{\{2,2\}}} \sqrt{\frac{d_{u}+d_{v}-2}{d_{u} \times d_{v}}} \\
& +\sum_{u v \in E_{\{2,4\}}} \sqrt{\frac{d_{u}+d_{v}-2}{d_{u} \times d_{v}}}+\sum_{u v \in E_{\{4,4\}}} \sqrt{\frac{d_{u}+d_{v}-2}{d_{u} \times d_{v}}} \\
=2 & \sqrt{\frac{2+2-2}{2 \times 2}+4(2 n-1) \sqrt{\frac{2+4-2}{2 \times 4}}+\left(6 n^{2}-8 n+2\right) 2 \sqrt{\frac{4+4-2}{4 \times 4}}} \\
= & \frac{2}{\sqrt{2}}+\frac{4(2 n-1)}{\sqrt{2}}+\left(6 n^{2}-8 n+2\right) \frac{\sqrt{6}}{4} \\
= & \sqrt{2}+2 \sqrt{2}(2 n-1)+\left(6 n^{2}-8 n+2\right) \frac{\sqrt{6}}{4} \\
= & \frac{4 \sqrt{2}+8 \sqrt{2}(2 n-1)+\sqrt{6}\left(6 n^{2}-8 n+2\right)}{4} \\
= & 3.6742 n^{2}+0.7578 n-0.1894 .
\end{aligned}
$$


(ii)

$$
\begin{aligned}
G A(\Gamma)= & \sum_{u v \in E(\Gamma)} \frac{2 \sqrt{d_{u} \times d_{v}}}{d_{u}+d_{v}} \\
& =\sum_{u v \in E\{2,2\}} \frac{2 \sqrt{d_{u} \times d_{v}}}{d_{u}+d_{v}}+\sum_{u v \in E\{2,4\}} \frac{2 \sqrt{d_{u} \times d_{v}}}{d_{u}+d_{v}} \\
& +\sum_{u v \in E\{4,4\}} \frac{2 \sqrt{d_{u} \times d_{v}}}{d_{u}+d_{v}} \\
& =\frac{2 \times 2 \sqrt{2 \times 2}}{2+2}+\frac{4(2 n-1) \times 2 \sqrt{2 \times 4}}{2+4}+\frac{\left(6 n^{2}-8 n+2\right) \times 2 \sqrt{4 \times 4}}{4+4} \\
& =2+\frac{16(2 n-1) \sqrt{2}}{6}+\frac{8\left(6 n^{2}-8 n+2\right)}{8} \\
& =2+\frac{8}{3}(2 n-1) \sqrt{2}+\left(6 n^{2}-8 n+2\right) \\
& =6 n^{2}-8 n+4+\frac{8 \sqrt{2}}{3}(2 n-1) \\
& =\frac{18 n^{2}-24 n+12+8 \sqrt{2}(2 n-1)}{3} \\
& =\frac{18 n^{2}-24 n+16 \sqrt{2} n+12-8 \sqrt{2}}{3} \\
& =6 n^{2}-0.4575 n+0.2287 .
\end{aligned}
$$


(iii)

$$
\begin{aligned}
A Z I(T)= & \sum_{u v \in E(T)}\left(\frac{d_{u} \times d_{v}}{d_{u}+d_{v}-2}\right)^{3} \\
& =\sum_{u v \in E_{\{2,2\}}}\left(\frac{d_{u} \times d_{v}}{d_{u}+d_{v}-2}\right)^{3}+\sum_{u v \in E_{\{2,4\}}}\left(\frac{d_{u} \times d_{v}}{d_{u}+d_{v}-2}\right)^{3} \\
& +\sum_{u v \in E_{\{4,4\}}}\left(\frac{d_{u} \times d_{v}}{d_{u}+d_{v}-2}\right)^{3} \\
& =2\left(\frac{2 \times 2}{2+2-2}\right)^{3}+4(2 n-1)\left(\frac{2 \times 4}{2+4-2}\right)^{3}+\left(6 n^{2}-8 n+2\right)\left(\frac{4 \times 4}{4+4-2}\right)^{3} \\
& =16+4(2 n-1) \times 8+\left(6 n^{2}-8 n+2\right)\left(\frac{8}{3}\right)^{3} \\
& =16+64 n-32+\left(6 n^{2}-8 n+2\right) \times 18.9629 \\
& =113.7774 n^{2}-87.7032 n+21.9258 .
\end{aligned}
$$

Theorem 4.4. Let $\Gamma \cong R H S L(n)$ be a rhombus oxide network with dimension $n$. Then, for $n \geq 2$, the fourth version of $\mathrm{ABC}\left(A B C_{4}\right)$, the fifth version of GA $\left(G A_{5}\right)$, and Sanskruti index (SI) are given as

(i) $A B C_{4}(\Gamma)=\frac{\sqrt{10}}{3}+\frac{8}{3 \sqrt{2}}+\sqrt{3}+4(2 n-3) \sqrt{\frac{5}{28}}+\frac{8}{\sqrt{7}}+2(4 n-7) \sqrt{\frac{13}{98}}+$ $\sqrt{8}(n-2)+6(n-2)^{2} \sqrt{\frac{15}{128}}$,

(ii) $G A(\Gamma)=2+\frac{4}{9} \sqrt{72}+\frac{2 \sqrt{96}}{5}+\frac{4 \sqrt{112}}{11}(2 n-3)+\frac{8}{13} \sqrt{168}+2(4 n-7)+$ $\frac{8 \sqrt{224}}{15}(n-2)+6(n-2)^{2}$,

(iii) $S(\Gamma)=3728.2699 n^{2}-5984.9639 n+2424.7359$.

Proof. Using Table 4 and the formulae defined in Section 2, we compute the required results as follow. 
(i)

$$
\begin{aligned}
& A B C_{4}=\sum_{u v \in E(\Gamma)} \sqrt{\frac{S_{u}+S_{v}-2}{S_{u} \times S_{v}}} \\
& =\sum_{u v \in E_{\{6,6\}}} \sqrt{\frac{S_{u}+S_{v}-2}{S_{u} \times S_{v}}}+\sum_{u v \in E_{\{6,12\}}} \sqrt{\frac{S_{u}+S_{v}-2}{S_{u} \times S_{v}}} \\
& +\sum_{u v \in E_{\{8,12\}}} \sqrt{\frac{S_{u}+S_{v}-2}{S_{u} \times S_{v}}}+\sum_{u v \in E_{\{8,14\}}} \sqrt{\frac{S_{u}+S_{v}-2}{S_{u} \times S_{v}}} \\
& +\sum_{u v \in E_{\{12,14\}}} \sqrt{\frac{S_{u}+S_{v}-2}{S_{u} \times S_{v}}}+\sum_{u v \in E_{\{14,14\}}} \sqrt{\frac{S_{u}+S_{v}-2}{S_{u} \times S_{v}}} \\
& +\sum_{u v \in E_{\{14,16\}}} \sqrt{\frac{S_{u}+S_{v}-2}{S_{u} \times S_{v}}}+\sum_{u v \in E_{\{16,16\}}} \sqrt{\frac{S_{u}+S_{v}-2}{S_{u} \times S_{v}}} \\
& =2 \sqrt{\frac{6+6-2}{6 \times 6}}+4 \sqrt{\frac{6+12-2}{6 \times 12}}+4 \sqrt{\frac{8+12-2}{8 \times 12}} \\
& +4(2 n-3) \sqrt{\frac{8+14-2}{8 \times 14}}+8 \sqrt{\frac{12+14-2}{12 \times 14}}+2(4 n-7) \sqrt{\frac{14+14-2}{14 \times 14}} \\
& +8(n-2) \sqrt{\frac{14+16-2}{14 \times 16}}+6(n-2)^{2} \sqrt{\frac{16+16-2}{16 \times 16}} \\
& =\frac{2}{6} \sqrt{10}+\frac{16}{\sqrt{72}}+4 \sqrt{\frac{18}{96}}+4(2 n-3) \sqrt{\frac{20}{112}} \\
& +8 \sqrt{\frac{24}{168}}+2(4 n-7) \sqrt{\frac{26}{196}}+8(n-2) \sqrt{\frac{28}{224}}+6(n-2)^{2} \sqrt{\frac{30}{256}} \\
& =\frac{\sqrt{10}}{3}+\frac{16}{6 \sqrt{2}}+4 \sqrt{\frac{3}{16}}+4(2 n-3) \sqrt{\frac{5}{28}}+8 \sqrt{\frac{1}{7}} \\
& +2(4 n-7) \sqrt{\frac{13}{98}}+8(n-2) \sqrt{\frac{1}{8}}+6(n-2)^{2} \sqrt{\frac{15}{128}} \\
& =\frac{\sqrt{10}}{3}+\frac{8}{3 \sqrt{2}}+\sqrt{3}+4(2 n-3) \sqrt{\frac{5}{28}} \\
& +\frac{8}{\sqrt{7}}+2(4 n-7) \sqrt{\frac{13}{98}}+\sqrt{8}(n-2)+6(n-2)^{2} \sqrt{\frac{15}{128}} .
\end{aligned}
$$


(ii)

$$
\begin{aligned}
& G A_{5}(\Gamma)=\sum_{u v \in E(\Gamma)} \frac{2 \sqrt{S_{u} \times S_{v}}}{S_{u}+S_{v}} \\
& =\sum_{u v \in E_{\{6,6\}}} \frac{2 \sqrt{S_{u} \times S_{v}}}{S_{u}+S_{v}}+\sum_{u v \in E_{\{6,12\}}} \frac{2 \sqrt{S_{u} \times S_{v}}}{S_{u}+S_{v}} \\
& +\sum_{u v \in E_{\{8,12\}}} \frac{2 \sqrt{S_{u} \times S_{v}}}{S_{u}+S_{v}}+\sum_{u v \in E_{\{8,14\}}} \frac{2 \sqrt{S_{u} \times S_{v}}}{S_{u}+S_{v}} \\
& +\sum_{u v \in E_{\{12,14\}}} \frac{2 \sqrt{S_{u} \times S_{v}}}{S_{u}+S_{v}}+\sum_{u v \in E_{\{14,14\}}} \frac{2 \sqrt{S_{u} \times S_{v}}}{S_{u}+S_{v}} \\
& +\sum_{u v \in E_{\{14,16\}}} \frac{2 \sqrt{S_{u} \times S_{v}}}{S_{u}+S_{v}}+\sum_{u v \in E_{\{16,16\}}} \frac{2 \sqrt{S_{u} \times S_{v}}}{S_{u}+S_{v}} \\
& =\frac{4 \sqrt{6 \times 6}}{6+6}+\frac{8 \sqrt{6 \times 12}}{6+12}+\frac{8 \sqrt{8 \times 12}}{8+12} \\
& +\frac{8(2 n-3) \sqrt{8 \times 14}}{8+14}+\frac{16 \sqrt{12 \times 14}}{12+14}+\frac{4(4 n-7) \sqrt{14 \times 14}}{14+14} \\
& +\frac{16(n-2) \sqrt{14 \times 16}}{14+16}+\frac{12(n-2)^{2} \sqrt{16 \times 16}}{16+16} \\
& =2+\frac{4}{9} \sqrt{72}+\frac{2 \sqrt{96}}{5}+\frac{4}{11}(2 n-3) \sqrt{112}+\frac{8}{13} \sqrt{168} \\
& +\frac{14(4 n-7)}{7}+\frac{8}{15}(n-2) \sqrt{224}+\frac{3}{8}(n-2)^{2} 16 \\
& =2+\frac{4}{9} \sqrt{72}+\frac{2 \sqrt{96}}{5}+\frac{4}{11}(2 n-3) \sqrt{112}+\frac{8}{13} \sqrt{168} \\
& +2(4 n-7)+\frac{8}{15}(n-2) \sqrt{224}+6(n-2)^{2} .
\end{aligned}
$$


(iii)

$$
\begin{aligned}
& S(\Gamma)=\sum_{u v \in E(\Gamma)}\left(\frac{S_{u} \times S_{v}}{S_{u}+S_{v}-2}\right)^{3} \\
& =\sum_{u v \in E_{\{6,6\}}}\left(\frac{S_{u} \times S_{v}}{S_{u}+S_{v}-2}\right)^{3}+\sum_{u v \in E_{\{6,12\}}}\left(\frac{S_{u} \times S_{v}}{S_{u}+S_{v}-2}\right)^{3} \\
& +\sum_{u v \in E_{\{8,12\}}}\left(\frac{S_{u} \times S_{v}}{S_{u}+S_{v}-2}\right)^{3}+\sum_{u v \in E_{\{8,14\}}}\left(\frac{S_{u} \times S_{v}}{S_{u}+S_{v}-2}\right)^{3} \\
& +\sum_{u v \in E_{\{12,14\}}}\left(\frac{S_{u} \times S_{v}}{S_{u}+S_{v}-2}\right)^{3}+\sum_{u v \in E_{\{14,14\}}}\left(\frac{S_{u} \times S_{v}}{S_{u}+S_{v}-2}\right)^{3} \\
& +\sum_{u v \in E_{\{14,16\}}}\left(\frac{S_{u} \times S_{v}}{S_{u}+S_{v}-2}\right)^{3}+\sum_{u v \in E_{\{16,16\}}}\left(\frac{S_{u} \times S_{v}}{S_{u}+S_{v}-2}\right)^{3} \\
& =2\left(\frac{6 \times 6}{6+6-2}\right)^{3}+4\left(\frac{6 \times 12}{6+12-2}\right)^{3}+4\left(\frac{8 \times 12}{8+12-2}\right)^{3} \\
& +4(2 n-3)\left(\frac{8 \times 14}{8+14-2}\right)^{3}+8\left(\frac{12 \times 14}{12+14-2}\right)^{3}+2(4 n-7)\left(\frac{14 \times 14}{14+14-2}\right)^{3} \\
& +8(n-2)\left(\frac{14 \times 16}{14+16-2}\right)^{3}+6(n-2)^{2}\left(\frac{16 \times 16}{16+16-2}\right)^{3} \\
& =93.312+364.5+606.815+702.464(2 n-3)+2744+856.797(4 n-7) \\
& +4096(n-2)+3728.270(n-2)^{2} \\
& =3728.270(n-2)^{2}+702.464(2 n-3)+856.797(4 n-7) \\
& +4096(n-2)+3808.627 \\
& =3728.2699 n^{2}-5984.9639 n+2424.7359 .
\end{aligned}
$$

\section{Comparison and Conclusion}

In this section, we compare the results of all the computed topological indices for the rhombus silicate and and rhombus oxide networks with the help of cartesian coordinate systems separately. 
In Section 3 and Section 4, the formulae of all the indices are found in term of $n$, where $n$ is dimension (number of vertices in any side) of the rhombus silicate or rhombus oxide network. In Figure 3, along the horizontal line the values of $n$ for silicate network are taken and along the vertical line the computed values of the indices are shown. We note that the values of the all the topological indices of the silicate network other than Sanskurti index (SI) and augmented Zagreb index (AZI) coincide. Similarly, in Figure 4 , the horizontal line shows the values of $n$ and the vertical line takes the computed values of the indices of the oxide network. The values of the all the topological indices of the oxide network other than Sanskurti index (SI) and augmented Zagreb index (AZI) coincide. Moreover, in both cases, all the topological indices remain constant approximately except Sanskurti index (SI) and augmented Zagreb index (AZI) with increasing values of $n$. The latest developed index SI shows a dominant change with the increasing value of $n$ for both the networks. Now, we close our discussion with the following lines.

In this paper, certain degree-based topological indices, namely general Randić, first general Zagreb, generalized Zagreb, multiplicative Zagreb, atom-bond connectivity (ABC), geometric-arithmetic (GA), augmented Zagreb, the fourth version of $\mathrm{ABC}\left(A B C_{4}\right)$, the fifth version of $\mathrm{GA}\left(G A_{5}\right)$ and Sanskruti indices of the rhombus silicate and rhombus oxide networks are studied and analytical closed formulas for these networks are determined that will help to understand the underlying topologies of these networks. 


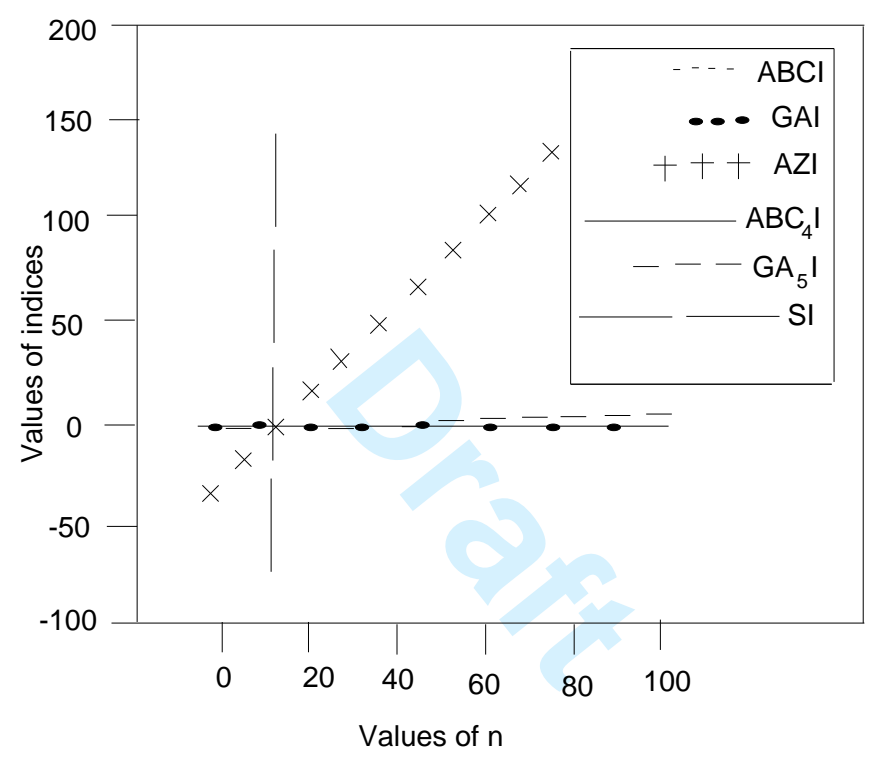

Figure 3: Comparison of the topological indices of the silicate network. 


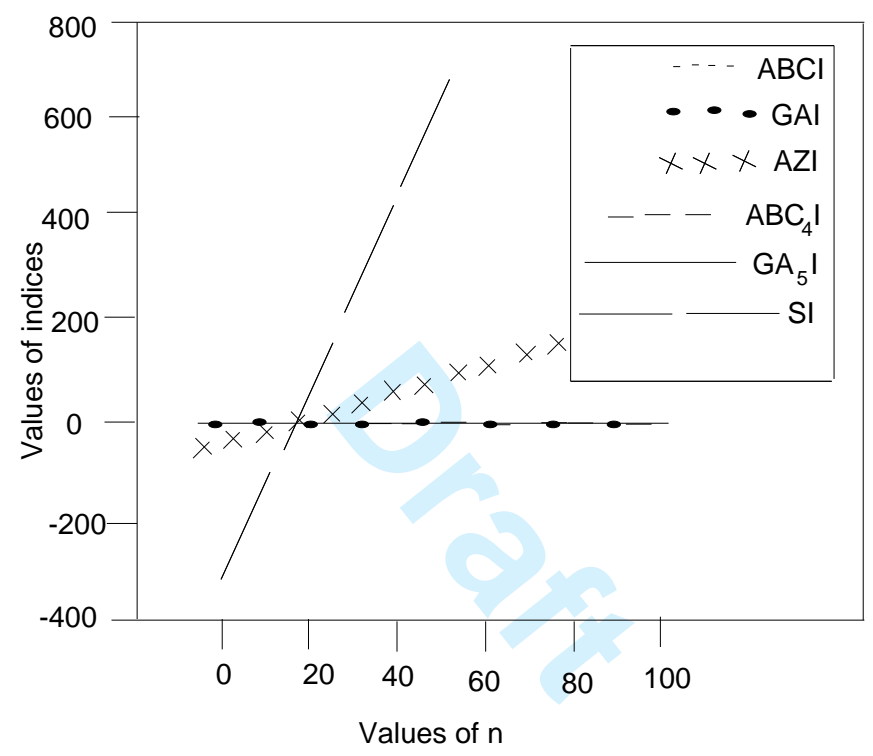

Figure 4: Comparison of the topological indices of the oxide network. 
Conflict of Interest: There is no conflict of interest in the publication of this paper.

Acknowledgement: The authors are indebted to the anonymous referees for their valuable comments to improve the original version of this paper. The first author is supported by Chinese Academy of Science Presidents International Fellowship Initiative (CAS-PIFI), Beijing, China. Grant No: 2015 PM 035 and the second author is supported CSC fellowship awarded by Chinese Scholarship Council. The third author is jointly supported by the National Science Foundation of China under Grants 61272530 and 61573096 . 


\section{References}

[1] Graovac, A.; Ghorbani, M.; Hosseinzadeh, M.A., J. Math. Nanosci., 1 (1)(2011) 33.

[2] Baig, A.Q.; Imran, M.; Ali, H., Can. J. Chem. 93(2015), 730.

[3] Akhter, A.; Imran, M., Can. J. Chem. 94(2016), 687.

[4] Imran, M.; Baig, A.Q.; Ali, H., J. Chemometrics 30(2016), 121.

[5] Bollobs, B,; Erdös, P., Ars Combin. 50 (1998) 225.

[6] Furtula, B.; Graovac, A.; Vukičević, D., J. Math. Chem. 48(2010), 370.

[7] Rajan, B.; William, A.; Grigorious, C.; Stephen, S., J. Comp. Math. Sci. Vol.3 5(2012), 530 .

[8] Amic, D.; Beslo, D.; Lucic, B.; Nikolic, S.; Trinajstić, N., J. Chem. Inf. Comput. Sci. 38 (1998) 819.

[9] Vukičević D.; Furtula, B., J. Math. Chem. 46(2009) 1369.

[10] Estrada, E.; Torres, L.; Rodríguez, L.; Gutman, I., Indian J. Chem. $37 \mathrm{~A}(1998), 849$.

[11] Britto Antony Xavier, G.; Suresh, E.; Gutman, I., Kragujevac Journal of Mathematics 38 1(2014), 95.

[12] Caporossi, G.; Gutman, I.; Hansen, P.; Pavlovíc, L., Comput. Biol. Chem. 27 (2003), 85.

[13] Wiener, H.J., J. Amer. Chem. Soc. 69 (1947), 17. 
[14] Gutman, I.; Trinajsti, N., Chem. Phys. Lett. 17(1972), 535.

[15] Gutman, I.; Polansky, O., Mathematical Concepts in Organic Chemistry, Springer-Verlag, Berlin, 1986.

[16] Devillers, J.; Balaban, A.T., Topological Indices and Related Descriptors in QSAR and QSPR, Gordon Breach, Amsterdam, 1999.

[17] Xu, K.; Das, K. Ch., Commun. Math. Comput. Chem. 68 (2012), 257.

[18] Azari, M.; Iranmanesh, A. Studia Univ. Babes-Bolyai. 56 (3) (2011), 59.

[19] Bača, M.; Horváthová, J.; Mokrišová, M.; and Suhányiová, A., Applied Mathematics and Computation 251(2015), 154.

[20] Bača, M.; Horváthová, J.; Mokrišová, M.; Semaničová-Feňovčková, A.; Suhńniov', A., Can. J. Chem. 93(2015), 1157.

[21] Ghorbani, M.; Azimi, N., Iran. J. Math. Chem. 3 (2)(2012), 137.

[22] Ghorbani, M.; Hosseinzadeh, M.A., Adv. Mater.-Rapid Commun. 4 (9)(2010), 1419.

[23] Randić M., J. Am. Chem. Soc. 97 (1975) 6609.

[24] Diudea M.V., QSPR/QSAR Studies by Molecular Descriptors, NOVA, New York, 2001.

[25] Hosamani, Sunilkumar M., J. Appl. Math. Comput. DOI 10.1007/s12190-016-1016-9.

[26] Li X.; Zheng, J., MATCH Commun. Math. Comput. Chem. 54(2005), 195. 
[27] Li, X.; Gutman, I., Mathematical Chemistry Monographs, No.1, Kragujevac, 2006.

[28] Hu, Y.; Li, X.; Shi, Y.; Xu, T.; Gutman, I., MATCH Commun. Math. Comput. Chem. 54(2005) 425. 